\title{
Enzyme-Supported Immunotherapy: Case Study and Possible Generalizations
}

\author{
Ulrich D. Jentschura \\ Department of Physics, Missouri University of Science and Technology, Rolla, MO, USA \\ Email: ulj@mst.edu
}

How to cite this paper: Jentschura, U.D. (2018) Enzyme-Supported Immunotherapy: Case Study and Possible Generalizations. Journal of Cancer Therapy, 9, 156-162. https://doi.org/10.4236/jct.2018.92016

Received: January 31, 2018

Accepted: February 23, 2018

Published: February 26, 2018

Copyright $\odot 2018$ by author and Scientific Research Publishing Inc. This work is licensed under the Creative Commons Attribution International License (CC BY 4.0).

http://creativecommons.org/licenses/by/4.0/

\section{(c) (i) Open Access}

\begin{abstract}
A combination therapy is discussed for the treatment of cancer, which has recently been applied successfully in a case study. The general approach is based on a combination of immune boosters, digestive enzymes and natural interferones. The idea is to stage a three-prong "pincer attack" on the tumor: while the immune boosters stimulate the immune system to attack cancer cells, the cytostatic properties of the interferones inhibit cancer growth, and the digestive enzymes accelerate the transport mechanism to remove the killed cancer cells from the body. The rationale of the therapy is explained, results of the case study are presented, and possible generalizations are mentioned.
\end{abstract}

\section{Keywords}

Immunotherapy, Digestive Enzymes, Natural Interferones, Active Surveillance, Withaferin A

\section{Introduction}

The purpose of the current paper is to describe a general approach to cancer therapy, which we would like to refer to as enzyme-supported immunotherapy, and which has recently been applied with good effect in a case study [1]. We observe that three different general approaches have been discussed separately in the literature before, namely, immunotherapy (support of the immune system in cancer treatment), interferones (which inhibit cancer cell growth), and digestive enzymes (which help the human body in getting rid of organic material which needs to be disposed of). However, a combination of these three approaches has never been described in the literature, to the best of our knowledge.

In order to put things into perspective, we note that immunotherapy is as old as $2600 \mathrm{BC}$, when a tumor found in pharaoh Djoser was bacterially infected by a 
physician by the name of Imhotep, using a poultice followed by an incision. Currently, interferones constitute a standard method in the treatment of cancer. Furthermore, digestive enzymes are an essential elements of the Gerson and Livingston-Wheeler therapies [2] [3], while both the Gerson and LivingstonWheeler therapies stress the importance of large doses of vitamin $\mathrm{C}$ in cancer treatment. The combination of the three ingredients (immunotherapy, interferones and digestive enzymes) has recently been described in Refs. [1] [4].

Here, we thus advocate a combination of the three approaches, sketched above, in the treatment of early-stage cancer, forming a so-called "pincer attack" on the tumor. The rationale behind the three-prong pincer attack is as follows. The immune boosters serve to put the immune system into "attack mode" and kill cancer cells, the interferones have cytostatic properties that inhibit cancer growth, while the digestive enzymes accelerate the transport mechanism to remove the killed cells, with the eventual aim that the killing rate of cancer cells exceeds the growth rate of the tumor.

\section{Methods and Summary of the Case Study}

The case study involved the author himself, who was diagnosed, in 2010, with a thyroid tumor. Details of the case study have been given in Ref. [1]; here, we provide a summary of the essential stages of the development of the diseasse and of the treatment. In particular, it should be mentioned that the case study involved a 45-year-old caucasian male without any appreciable diseases except the thyroid tumor.

Despite repeated percutaneous ethanol injections (PEI) and a $30 \mathrm{mCi}$ dose of 131 I (given in 2013), a (probably initially benign) tumor of the thyroid grew, and biopsies revealed primary and secondary mutations in cells, typical of thyroid carcinoma (in 2014). Specifically, in 2014, the morphological analysis of a biopsy indicated a microfollicular Hurthle cell pattern, trabecular groups, complex 3D groups, bubble gum colloids and giant cells, swirl patterns and abnormal microfollicular patterns. These indicated possible secondary changes in the cells due to thyroid carcinoma. The biopsied cells also failed several RNA translocation tests, such as RET-PTC1, RET-PTC3, and PAX8-PPAR $\gamma$. In 2014, self-palpitation revealed that the thyroid mass had changed from a soft-tissue goiter to a rather hard mass. In all, the result of the biopsy was consistent with the follicular variant of papillary thyroid carcinoma. The size of the tumor was estimated as $80 \mathrm{ml}$ under ultrasound imaging.

An intense enzyme-supported immunotherapy was begun in a trial involving one single subject (the author himself), with the following ingradients:

- An immune booster (vitamin $\mathrm{C}$, enteric coated) was administered in a dosage of $3000 \mathrm{mg}$ per day. It was taken together with a vitamin A and B6-B12 complex (one tablet per day each, of a generic off-the-shelf product, details were not monitored). Furthermore, echinacea was applied (two tablets per day, $600 \mathrm{mg})$. 
- An interferone (ashwaghanda, extract of withaferania somnia) which contains the interferone withaferin $\mathrm{A}$, was administered in a dosage of 4 tablets per day, amounting to about $1200 \mathrm{mg}$ ashwaghanda root extract. This was supplemented by about 2 daily tablets of kachnar (bauhinia tormentosa) corresponding to about $600 \mathrm{mg}$ of bark extract per day.

- Digestive enzymes (Serratiopeptidase) in a dose of 360,000 SPUs per day, corresponding to 3 tablets each with 120,000 SPU.

Almost complete remission of the thyroid mass was observed in the case study, after the continued application of the combination therapy over a time frame of about 18 months. The size of the tumor was reduced from about $80 \mathrm{ml}$ to an estimated $12 \mathrm{ml}$ for the total size of the left thyroid lobe, under ultrasound imaging. Concomitantly, the tumor became soft, all cancer markers were determined as negative under a repeat biopsy, and the entire left thyroid lobe became unsuspicious under ultrasound imaging. We note that a total size of 12 $\mathrm{ml}$ is consistent with an enlarged, but not clinically relevant, thyroid lobe. Again, further details can be found in Refs. [1] [4]. In the case study, therapy has been discontinued for several weeks in late 2017 without any adverse effects. However, in order to prevent recurrence of the tumor, and in order to address possible long-term consequences of the (unsuccessful) application of radiation therapy in the case study, a continuous prophylactic regimen involving small doses of the active ingredients (300 mg of kachnar bark extract and $300 \mathrm{mg}$ of ashwaghanda per day) is currently being continued.

While caffeine and taurine consumption may have played a role in goitrogenesis, as described in detail in Refs. [1] [4], the remission of the tumor should be attributable to the three-prong pincer attack treatment, as no other factors were involved during the remission process.

\section{Results, Implications and Interpretation}

In order to understand the context of the case study [1], it is useful to remember the followingfacts:

- Immune boosters are important in the context of immuntherapy. E.g., at the VEPP-4M accelerator of the Academy Institute of the Russian Federation in Novosibirsk, radiation therapy on cancer is accompanied by short-term immune booster therapy which serves to drive out the killed or weakened cancer cells [5]. This wisdom has been a significant motivating factor in the trial application of the above mentioned combined enzyme-immunotherapy. Immune augmentation using Anti-CTLA-4 antiboies has also been advocated within cancer treatment [6].

- Concerning natural interferones, it is surprising that the mechanism behind the conjectured clinical relevance of certain ayurvedic medicine has recently been studied on the molecular level. E.g., several studies have confirmed cytostatic properties of withaferin A, the interferon contained in ashwaghanda [7] [8] [9] [10]. 
- Digestive enzymes have long been an essential accepted element in Asian (Japanese) medicine. Serratiopeptidase (Serrapeptase) constitutes a digestive enzyme that eliminates cell material very effectively and is used by the Chinese silk worm during metamorphosis (i.e., during the break-up of the caterpillar's cocoon). Typically, the Chinese silk worm is able to digest a parachute-quality silk cocoon of the silk worm larva in 15 minutes.

In the case study [1] [4], remission of a hardened $80 \mathrm{ml}$ tumor with a suspicious biopsy and failed RNA translocations tests in the left lobe of the thyroid to a soft, $12 \mathrm{ml}$ thyroid lobe with an unsuspicious ultrasound image, unsuspicious RNA translocation tests and negative cancer markers could be observed. We recall, once more, the dosage for cancer therapy, which has been optimized in the case study [1] through quite intense self-experimentation:

- Vitamin C, enteric coated, in a dosage of $3000 \mathrm{mg}$ per day.

- Vitamin A and B6-B12 complex according to RDA.

- Echinacea (two tablets per day, $600 \mathrm{mg}$ ).

- Ashwaghanda root extract (1200 mg per day).

- Kachnar (bauhinia tormentosa) tree bark extract (600 mg per day).

- Serratiopeptidase (360,000 SPUs per day).

Concerning the onset of thyroid disease in the case study [1], we would briefly like to discuss the possible role of environmental factors in cancerogenesis, which in the particular case study [1] refers to an increased intake in caffeine and taurine. Namely, according to Ref. [11], numerous epidemiological studies have established strong evidence that many cancers have risk proportions attributed to environmental exposures. This is supported, according to Ref. [11], by the fact that order-of-magnitude international geographical variations exist in the incidence rates (for example, in breast and prostate cancers). More interestingly, immigrants moving from countries with lower cancer incidence to countries with higher cancer rates soon acquire the higher risk of their new country [12] [13]. Even if several risk factors have been identified for these cancers, it is remarked in Ref. [11] that no single one can account for their substantial extrinsic risk proportions. The authors of Ref. [11] also observe that at least $75 \%$ of oesophageal cancer, or head and neck cancer cases, are caused by tobacco and alcohol (see also Refs. [14] [15]).

The case study [1] suggests that large doses (close to the maximum RDA) of caffeine and taurine cannot be excluded as goitrogens. These observations support the conclusions of Ref. [11], and provide further evidence for the conjecture that environmental factors play important roles in cancer incidence, and, furthermore, that they are modifiable through lifestyle changes and/or vaccinations. A possible mechanism explaining the role of taurine in goitrogenesis, bypassing the thyroid-stimulating hormone (TSH), has been described in Ref. [1]. In a rat model, co-promoting effects of high doses of caffeine in thyroid carcinogenesis have been described [16], while the role of caffeine in human thyroid carcinogenesis remains controversial [17].

Specifically, one might ask: How could caffeine and/or taurine possibly 
contribute to thyroiditis, or thyroid carcinogenesis, if they do not lead to increased levels of thyroid stimulating hormone? By what mechanism could they "bypass" the ordinary stimulation mechanism?

A hint might be given in in an animal (rat) model [18], where taurine has been shown to enhance T4 to T3 conversion. It is generally estimated that about $10 \%-20 \%$ of the human $\mathrm{T} 4$ to $\mathrm{T} 3$ conversion proceeds in the thyroid itself. A combination of caffeine and taurine is typically contained in so-called "energy drinks". The enhanced level of physical and mental activity associated with energy drinks, in principle, is a desirable outcome of their consumption. Let us assume that the findings of the rat model [18] are applicable to humans. In this case, the direct stimulation of T4 to T3 conversion by taurine, together with the enhancement of physical activity by caffeine, could provide a mechanism for bypassing the "ordinary" TSH mechanism for thyroid stimulation.

\section{Conclusions and Perspectives}

We have discussed enzyme-supported immunotherapy as a combination therapy for a malignant carcinoma, based on a combination of immune boosters, natural interferones and digestive enzymes. The combination of cancer cell growth inhibitors combined with immune boosters and digestive enzymes may lead to a natural interplay of all of these ingredients.

The effectiveness of the therapy has been subject to a case study [1] [4]. Even if the results of the case study [1] support possible clinical benefits of enzymesupported immunotherapy, one should be very careful and stressful that the new therapy could also assist, not replace, traditional forms of treatment such as radiation therapy, chometherapy, and surgery. Furthermore, one may point out that the therapy has been applied to a (very likely) stage I carcinoma. It remains to be seen if it can be applied to stage II, III and IV carcinomas.

One may envisage generalizations of the approach, based on

- the intravenous administration of large doses of immune boosters in the initial phase of treatment,

- higher doses of natural interferones in the initial phase,

- conceivably combined with the injection of digestive enzymes directly into the tumor,

Furthermore, one may consider replacing the unspecific activation of the immune systems by a more specific one, depending on the particular case at hand. In the Livingston-Wheeler therapy, emphasis is laid on antibodies against progenitor cryptocides, a bacterium conjectured to play a decisive and universal role in carcinogenesis [19] [20]. Of course, such a specific activation would not be effective in cases where environmental factors and detrimental nutritional habits [1] [11] are decisive factors in carcinogenesis.

\section{Acknowledgements}

The development of the therapy method described in this article was influenced 
by the same logic which is routinely applied to selected problems in theoretical physics; NSF (National Science Foundation) grants PHY-1403973 and PHY1710856 are gratefully acknowledged, as well as helpful conversations with A. Kleppe, A. I. Milstein and R. B. Guttler.

\section{References}

[1] Jentschura, U.D. (2017) Thyroid Disease and Taurine Consumption: A Case Study on Enzyme-Supported Immunotherapy. U.S. Patent Pending 62/577,284.

https://doi.org/10.6084/m9.figshare.5828058

[2] Livingston-Wheeler, V. and Wheeler, O.W. (1980) The Microbiology of Cancer: Physician's Handbook. Livingston-Wheeler Medical Clinic, San Diego, CA.

[3] Gerson, M. (2002) A Cancer Therapy: Results of Fifty Cases. Gerson Institute, San Diego, CA.

[4] Jentschura, U.D. (2017) U.S. Patent Pending 62/577,284.

[5] Milstein, A.I. (2007) Private Communication.

[6] Chen, J. and Hill, C. (2012) U.S. Patent No. 8,226,946.

[7] Thaiparambil, J.T., Bender, L., Ganesh, T., Kline, E., Patel, P., Liu, Y., Tighiouart, M., Vertino, P.M., Harvey, R.D., Garcia, A. and Marcus, A.I. (2011) Withaferin A Inhibits Breast Cancer Invasion and Metastasis at Sub-Cytotoxic Doses by Inducing Vimentin Disassembly and Serine 56 Phosphorylation. International Journal of Cancer, 129, 2744-2755. https://doi.org/10.1002/ijc.25938

[8] Choi, B.Y. and Kim, B.-W. (2015) Withaferin-A Inhibits Colon Cancer Cell Growth by Blocking STAT3 Transcriptional Activity. Journal of Cancer Prevention, 20, 185-192. https://doi.org/10.15430/JCP.2015.20.3.185

[9] Lee, I.-C. and Choi, B.Y. (2016) Withaferin-A-A Natural Anticancer Agent with Pleitropic Mechanisms of Action. International Journal of Molecular Sciences, 17, 290. https://doi.org/10.3390/ijms17030290

[10] Palliyaguru, D.L., Singh, S.V. and Kensler, T.W. (2016) Withania somnifera: From Prevention to Treatment of Cancer. Molecular Nutrition \& Food Research, 60, 1342-1353. https://doi.org/10.1002/mnfr.201500756

[11] Wu, S., Powers, S., Zhu, W. and Hannun, Y.A. (2016) Substantial Contribution of Extrinsic Risk Factors to Cancer Development. Nature, 529, 43-47.

https://doi.org/10.1038/nature16166

[12] Gray, J., Evans, N., Taylor, B., Rizzo, J. and Walker, M. (2009) State of the Evidence: The Connection between Breast Cancer and the Environment. International Journal of Occupational and Environmental Health, 15, 43-78.

[13] Shimizu, H., Ross, R.K., Bernstein, L., Yatani, R., Henderson, B.E. and Mack, T.M. (1991) Cancers of the Prostate and Breast among Japanese and White Immigrants in Los Angeles County. British Journal of Cancer, 63, 963-966.

https://doi.org/10.1038/bjc.1991.210

[14] Blot, W.J., McLaughlin, J.K., Winn, D.M., Austin, D.F., Greenberg, R.S., PrestonMartin, S., Bernstein, L., Schoenberg, J.B., Stemhagen, A. and Fraumeni, J.F. (1988) Smoking and Drinking in Relation to Oral and Pharyngeal Cancer. Cancer Research, 48, 3282-3287.

[15] Kamangar, F., Chow, W.-H., Abnet, C. and Dawsey, S. (2009) Environmental Causes of Esophageal Cancer. Gastroenterology Clinics of North America, 38, 27-57. https://doi.org/10.1016/j.gtc.2009.01.004 
[16] Son, H.-W., Nishikawa, A., Okazaki, K., Kitamura, Y., Kanki, K., Lee, K.-Y., Umemura, T. and Hirose, M. (2004) Specificity of Co-Promoting Effects of Caffeine on Thyroid Carcinogenesis in Rats Pretreated with N-Bis(2-hydroxypropyl)nitrosamine. Toxicologic Pathology, 32, 338-344. https://doi.org/10.1080/01926230490431853

[17] Han, M.A. and Jim, J.H. (2017) Coffee Consumption and the Risk of Thyroid Cancer: A Systematic Review and Meta-Analysis. International Journal of Environmental Research and Public Health, 14, 129 (Article No., 9 pages).

[18] Zhang, J., Huang, S.H. and Chen, B.M. (2002) Effect of Taurine on Thyroid Hormone and Second Messenger in Myocardium of Rats after Exhaustive Exercise, Chinese Journal of Applied Physiology (in Chinese: Zhongguo Ying Yong Sheng Li Xue Za Zhi), 18, 159-161.

[19] Livingston-Wheeler, A.M., Livingston-Wheeler, V.W. and Alexander-Jackson, E. (1970) Toxic Fractions Obtained from Tumor Isolates and Related Clinical Implications. Annals of the New York Academy of Sciences, 174, 675-689. https://doi.org/10.1111/j.1749-6632.1970.tb45590.x

[20] Livingston-Wheeler, V.W. and Livingston-Wheeler, A.M. (1974) Some Cultural, Immunological, and Biochemical Properties of Progenitor Cryptocides. Transactions of the New York Academy of Sciences, 36, 569-582. 